\title{
Three-phase unbalanced compensation and its application in transformer region based on Wang's theorem
}

\author{
Baoxuan Ye ${ }^{\mathrm{a}}$, Yuwu Wu ${ }^{\mathrm{a}}$, Bingzhang Tong ${ }^{\mathrm{a}}$, Kangjian Wang ${ }^{\mathrm{a}}$, Luqing Wang ${ }^{\mathrm{a}}$, \\ Xiangmin Huang \\ ${ }^{a}$ Hainan power grid Co., Ltd,Wenchang 571300, Hainan, China \\ ${ }^{b}$ BENLIU Electrical Technology Co., Ltd, Guangzhou 510640, Guangdong, China
}

\begin{abstract}
The passive component compensation for three-phase unbalance treatment at this stage is difficult to adapt to the load fluctuations and rapid changes in actual distribution network. Therefore, aiming at the goal of active power balance, firstly, the paper derives the formula of susceptance compensation among phases based on the Wang's theorem. Next, correct the basic formula and verify it by simulation, considering engineering factors such as actual line impedance, power supply asymmetry and so on. Finally, summarize a relatively complete configuration method of three-phase unbalance compensation in transformer region. The results show that the method can effectively improve the adaptability of the passive component compensation.
\end{abstract}

Keywords: three-phase unbalance; Wang's theorem; passive component compensation; engineering application

\section{Introduction}

Three-phase unbalance is one of the important indicators to measure power quality. If the problem in a distribution network is serious, it means that there is a large energy loss and voltage drop in the network to a certain extent. To solve the problem, in recent years, scholars around the world have carried out a great deal of research on the control measures of three-phase unbalance. The main methods are phase order equilibrium and load compensation [1,2], the former must strictly consider the grid structure and load sensitivity [3-5], so the latter is more common [6-7]. Steinmetz first proposed the theory of ideal compensation network [8]; the article [9] proposed to decompose unbalanced current with symmetric component method. The article [10] proposed the instantaneous power theory (pq theory), which can be used to simultaneously compensate for reactive power, negative sequence, harmonic current, etc. As for the current device of load compensation, the cost of power electronics device is relatively high, which is only used in medium and high voltage distribution network, so the passive component device has become the main tool. However, most of previous researches of it neglect the influence of engineering factors of it, so the effect is not as good as expected. This paper is based on Wang's theorem and studies the threephase unbalance compensation configuration method, which has a strong adaptability.

\section{Derivation of Susceptance Compensation Based on Wang's Theorem}

\subsection{The basic principle of Wang's theorem}

Considering that active power is an average of the integral of instantaneous power that the supply emits or the load consumes in a cycle, so it can get rid of the influence of instantaneous current and voltage values, also weaken the impact of load fluctuation to a certain extent. Therefore, this paper

\footnotetext{
* Manuscript received March 15, 2018; revised July 19, 2019.

Corresponding author: Tel.: +86-18826076283; E-mail address: $1870453691 @ q q . c o m$.

doi: $10.12720 /$ sgce.8.5.574-579
} 
proposes a three-phase unbalance compensation method for engineering applications, from the aspect of active power transfer. The principle the paper adapts is one of Wang's theorems: "Capacitors or inductors across two phases can transfer active power between two phases," as is shown in Fig. 1.

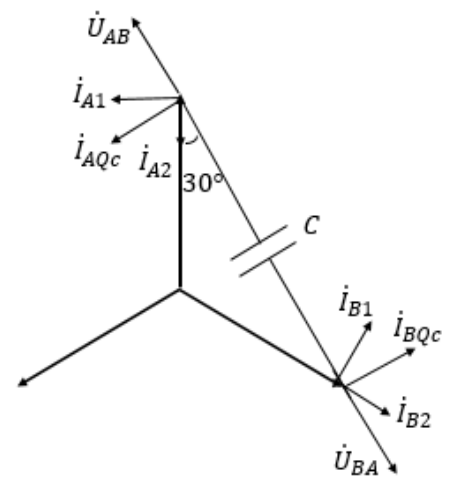

Fig. 1. Vector graph in parallel with capacitance between two phases

\subsection{Calculation formula of susceptance compensation}

Wang's theorem qualitatively analyzes the power transfer effect of each parallel component, now the paper quantitatively analyzes the effect by deducing the phase compensation susceptance calculation formula and correcting it after taking full account of the actual engineering factors.

According to Wang's theorem, if the three-phase voltage is symmetrical and the amplitude is equal, as we can see in Fig. 1, the active power transferred of capacitance in parallel is:

$$
\Delta P=U_{A} \cdot I_{A Q c} \cdot \cos 60^{\circ}=\sqrt{3} / 2 \omega C U^{2}
$$

In the formula, $\omega$ : Sinusoidal AC electrical angular frequency; $C$ : Capacity of parallel capacitor; $U$ : Three-phase voltage amplitude.

If the active power of two capacitors in parallel is $P_{1}$ and $P_{2}$, and $P_{1}>P_{2}$, in order to equalize two phases' active power, there should be: .

$$
C=2 \Delta P / \sqrt{3} w U^{2}=\left(P_{1}-P_{2}\right) / \sqrt{3} w U^{2}
$$

Similarly, if there is inductance in parallel, formulas should be:

$$
\begin{gathered}
\Delta P=\sqrt{3} U^{2} / 2 \omega L \\
L=\sqrt{3} U^{2} / 2 \omega \Delta P=\sqrt{3} U^{2} / \omega\left(P_{1}-P_{2}\right)
\end{gathered}
$$

\section{Correction of the Formula}

According to the wiring diagram in Fig. 2, the three-phase load asymmetric simulation model is set up based on Simulink simulation platform as Fig 3, which consists of three-phase symmetrical power supply, low voltage distribution network wire model, adjustable load impedance and package measurement Subsystem, the voltage amplitude of the power supply is $220 \mathrm{~V}$, the total length of the line is $0.5 \mathrm{~km}$. 


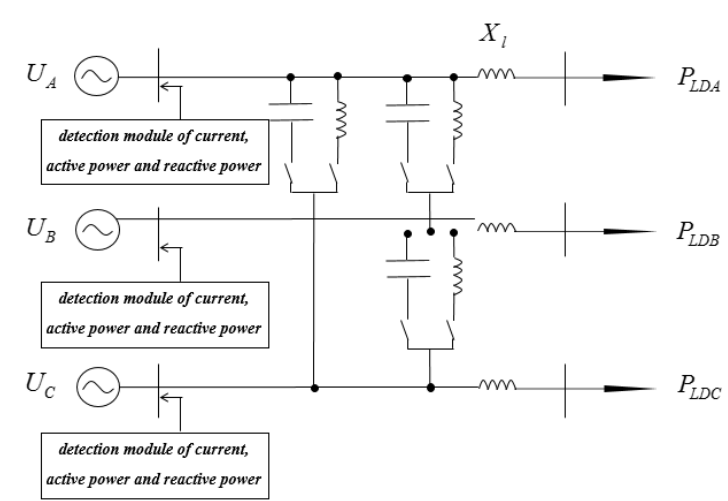

Fig. 2. Wiring diagram

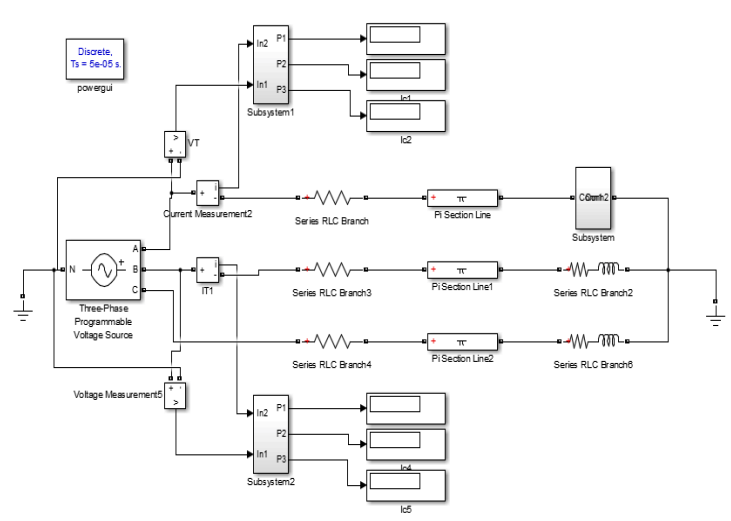

Fig. 3. Simulation model with three-phase unbalanced

\subsection{Simulation analysis of the ideal Line impedance model}

Neglecting the line impedance between the capacitor in parallel and the distribution transformer respectively set the load impedance of each phase as follows:

Situation 1): $Z_{A}=0.389+j 0.241 \Omega, Z_{B}=1.166+j 0.722 \Omega, Z_{C}=1.166+j 0.722 \Omega$

Situation 2): $Z_{A}=1.166+j 0.722 \Omega, Z_{B}=1.166+j 0.722 \Omega, Z_{C}=1.166+j 0.722 \Omega$

Calculate the compensation capacitance of phase A and B according to (2), the simulation parameters obtained before and after connecting capacitance between two phases are as shown in Table 1 and Table 2 .

Table 1. Data comparison situation 1)

\begin{tabular}{ccccc}
\hline \multirow{2}{*}{ data } & \multicolumn{2}{c}{$\mathrm{P}(\mathrm{kW})$} & \multicolumn{2}{c}{$\mathrm{Q}(\mathrm{kVar})$} \\
& Phase A & Phase B & Phase A & Phase B \\
\hline Before & 60.29 & 25.69 & 38.90 & 16.19 \\
After & 43.07 & 42.89 & 9.112 & -13.6 \\
\hline
\end{tabular}

Table 2. Data comparison situation 2)

\begin{tabular}{ccccc}
\hline \multirow{2}{*}{ Data } & \multicolumn{2}{c}{$\mathrm{P}(\mathrm{kW})$} & \multicolumn{2}{c}{ Q(kVar) } \\
& Phase A & Phase B & Phase A & Phase B \\
\hline Before & 25.69 & 60.29 & 16.19 & 38.9 \\
After & 8.485 & 77.49 & -13.6 & 9.112 \\
\hline
\end{tabular}

The active powers of two phases are basically equal in Table 1, but completely different in Table 2, indicating that the phase order should be considered when applying Eq. (2) and Eq. (4).

\subsection{Simulation analysis of asymmetric power supply}

Simulation analysis above is carried out under the assumption of symmetrical power supply. If the three-phase power supply is asymmetrical, (1) and (3) should be respectively revised as follows:

$$
\Delta \mathrm{P}=\omega \mathrm{C} U_{1} U_{2} \sin \varphi_{12}(5) \Delta \mathrm{P}=U_{1} U_{2} \sin \varphi_{12} / \omega L
$$

Correspondingly, (2) and (4) should be corrected as:

$$
\mathrm{C}=\left(P_{1}-P_{2}\right) / \omega U_{1} U_{2} \sin \varphi_{12}
$$

$$
\mathrm{L}=U_{1} U_{2} \sin \varphi_{12} / \omega\left(P_{1}-P_{2}\right)
$$

In addition, the incremental formula of reactive power can be deduced as:

$\Delta Q_{1 C}=\omega C U_{1} U_{12} \cos \alpha_{1}$

$$
\cos \alpha_{1}=\sqrt{1-\left(U_{2} \sin \varphi_{12} / U_{12}\right)^{2}}
$$

$$
\begin{array}{r}
\Delta Q_{1 L}=U_{1} U_{12} \cos \alpha_{1} / \omega L \\
U_{12}=\sqrt{U_{1}^{2}+U_{2}^{2}-2 U_{1} U_{2} \cos \varphi_{12}}
\end{array}
$$

Set the amplitudes of supply are $240 \mathrm{~V}, 200 \mathrm{~V}$ and $220 \mathrm{~V}$ respectively but the phases still symmetrical, load impedances are as follow: $Z_{A}=0.389+j 0.241 \Omega, Z_{B}=1.166+j 0.722 \Omega, Z_{C}=1.166+j 0.722 \Omega$;

The capacity of capacitance in parallel is set as $1941 \mu \mathrm{F}$ according to Eq. (7), and the theoretical value and calculated value of active power and reactive power increment are shown in the table 3 . 
Table 3. Data comparison when the power supply is asymmetrical

\begin{tabular}{ccccc}
\hline Data & $P_{A}(\mathrm{~kW})$ & $P_{B}(\mathrm{~kW})$ & $\Delta Q_{A}(\mathrm{kVar})$ & $\Delta Q_{B}(\mathrm{kVar})$ \\
\hline Before & 71.98 & 21.35 & - & - \\
After(theoretical) & 46.665 & 46.665 & 49.656 & 39.000 \\
After(calculated) & 46.65 & 46.67 & 49.63 & 38.99 \\
Error (\%) & 0.032 & 0.011 & 0.052 & 0.026 \\
\hline
\end{tabular}

It can be seen from Table 3 that the configuration of the capacitance between two phases with asymmetrical power supply should be adjusted according to (7).

\subsection{Simulation analysis considering line impedance}

Now consider the impact of actual line impedance. Suppose the power supply is symmetrical and voltage amplitude is $220 \mathrm{~V}, Z_{A}=0.389+j 0.241 \Omega, Z_{B}=Z_{C}=1.166+j 0.722 \Omega$; The capacity of the capacitance in parallel is set according to (5). The result is shown in Table 4.

Table 4. Effect of the position where capacitance parallel on active power balance

\begin{tabular}{ccc}
\hline The parallel position away from the transformer & Phase A & Phase B \\
\hline (Before) & 60.29 & 25.69 \\
0 & 43.07 & 42.89 \\
300 meters & 48.94 & 41.35 \\
500 meters & 51.98 & 39.59 \\
\hline
\end{tabular}

It can be seen from Table 4 that when the capacitance is connected to the low-voltage side of distribution transformer, the best effect of active power balance is best.

\section{Configuration Method of Three-phase Unbalance Compensation}

Combined with the above three simulations, based on the superposition theorem, a set of relatively perfect three-phase imbalance compensation configuration method for engineering applications is proposed, as shown in the flow chart of Fig. 4.

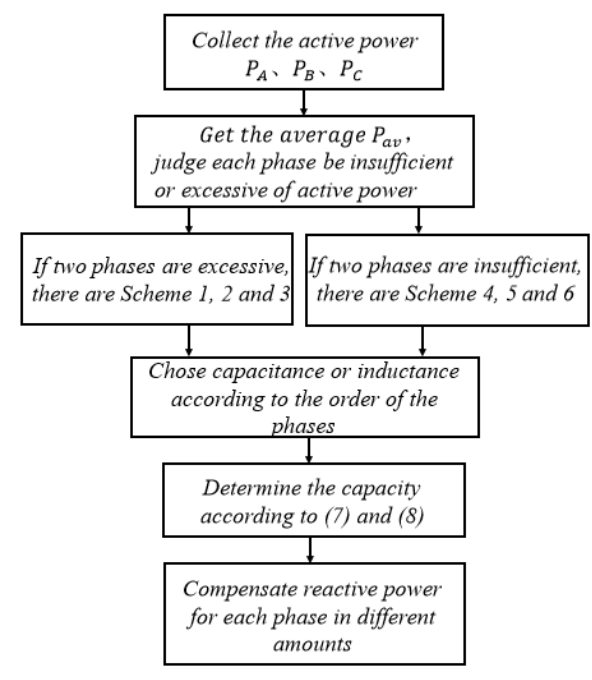

Fig.4. Configuration flow chat

The method in the flow chart to judge each phase insufficient or excessive of active power is as follow: Use Eq. (13) to calculate, if $\Delta P_{i}<0$, the active power is insufficient, else is excessive.

$$
\Delta P_{i}=P_{i}-P_{\text {av }}(\mathrm{i}=A, B, C)
$$


If two of the three phases are excessive of active power and one is insufficient, then the corresponding phases are set as $X_{1}, Y_{1}$ and $Z_{1}$. Then the directions the active power transfers of Schemes 1, 2 and 3 in the flowchart are as follow:

Schemes 1: $X_{1} \rightarrow Z_{1}, \quad Y_{1} \rightarrow Z_{1}$; Schemes 2: $X_{1} \rightarrow Y_{1}, \quad Y_{1} \rightarrow Z_{1}$; Schemes 3: $Y_{1} \rightarrow X_{1}, X_{1} \rightarrow Z_{1}$

If two of the three phases are insufficient of active power and one of the three phases is excessive, then the corresponding phases are set as $X_{2}, Y_{2}$ and $Z_{2}$. Then the directions the active power transfers of Schemes 4, 5 and 6 in the flowchart are as follow:

Schemes 4: $Z_{2} \rightarrow X_{2}, Z_{2} \rightarrow Y_{2}$; Schemes 5: $Z_{2} \rightarrow X_{2}, X_{2} \rightarrow Y_{2}$; Schemes 6: $Y_{1} \rightarrow X_{1}, X_{1} \rightarrow Z_{1}$

Tip: As the load is generally inductive, the solution that two inductances in parallel is not accepted.

\section{Simulation Verification}

Set the parameters of the simulation model with three-phase unbalanced in Fig. 6. Keep the power supply to be symmetry and the amplitude is $220 \mathrm{~V}$, the total length of the wire is $0.5 \mathrm{~km}$. $Z_{A}=0.389+j 0.241 \Omega, Z_{B}=Z_{C}=1.166+j 0.722 \Omega$, Now follow the steps of Fig. 4 to configure:

- Collect the average of each phase's active power in the low-voltage side of the distribution transformer and set them as $P_{A}, P_{B}$ and $P_{C}$, which are respectively $82.51 \mathrm{~kW}, 25.69 \mathrm{~kW}$ and $45.11 \mathrm{~kW}$.

- Get the average $P_{a v}=51.1 \mathrm{~kW}$ of the three phases, what the result indicates is:

Phase A is excessive because $\Delta P_{A}=31.41 \mathrm{~kW}$; Phase B is insufficient because $\Delta P_{B}=-25.41 \mathrm{~kW}$; Phase $\mathrm{C}$ is insufficient because $\Delta P_{C}=-5.99 \mathrm{~kW}$.

- Phase $\mathrm{B}$ and $\mathrm{C}$ are insufficient of the active power and phase $\mathrm{A}$ are excessive, so the options are scheme 4, 5 and 6 . According to the order of the phases, three of the scheme are as follow:

Scheme 4: Phase A and B are in parallel with capacitance and phase A and C are parallel with inductance;

Scheme 5: Phase A and B are in parallel with capacitance and phase B and C are parallel with capacitance;

Scheme 6: Phase A and $\mathrm{C}$ are in parallel with inductance and phase $\mathrm{B}$ and $\mathrm{C}$ are parallel with inductance;

Among them, the scheme 6 is given up because of two inductances in parallel.

- Calculate the capacity of the capacitance and inductance, then:

Scheme 4: $\Delta P_{A B}=25.41 \mathrm{~kW}, \Delta P_{A C}=5.99 \mathrm{~kW}, C_{A B}=1.95 \mathrm{mF}, L_{A C}=22.274 \mathrm{mH}$;

Scheme 5: $\Delta P_{A B}=31.41 \mathrm{~kW}, \Delta P_{B C}=5.99 \mathrm{~kW}, C_{A B}=2.387 \mathrm{mF}, C_{B C}=0.455 \mathrm{mF}$.

- Compensate reactive power for each phase in different amounts. Aiming at the power factor as 1 , then the components to be bridged between each phase and the zero line of two schemes are as follow:

Scheme 4: $C_{A}=1.326 m F, L_{B}=5.50 m H, C_{C}=2.58 m F$;

Scheme 5: $L_{A}=2.19 H, L_{B}=3.195 m H, C_{C}=1.22 m F$.

- Comparison of the active power before and after compensation are shown in Table 5,

Table 5. Data comparison before and after compensating

\begin{tabular}{cccc}
\hline Data & $P_{A}(\mathrm{~kW})$ & $P_{B}(\mathrm{~kW})$ & $P_{C}(\mathrm{~kW})$ \\
\hline Before & 82.51 & 25.69 & 45.11 \\
After (Scheme 4) & 51.26 & 50.97 & 51.07 \\
After (Scheme 5) & 51.23 & 51 & 51.06 \\
\hline
\end{tabular}

It can be seen from Table 5 that the configuration of the above two schemes can balance active power and the three-phase unbalanced compensation practical configuration are effective.

\section{Conclusion}

Based on the Wang's theorem, this paper fully considers the factors such as the actual circuit model, asymmetric power supply and other factors, the formula of susceptance compensation among phases 
considering the active power balance is obtained, and a more complete configuration method of threephase unbalance compensation in transformer region is proposed and verified by simulation. This configuration method makes up for the shortcomings of the previous studies in practical engineering applications and has strong adaptability to load fluctuation.

\section{References}

[1] Zhang YJ, Shi H, Xu L. Systematic developing program of distribution networks energy saving potential evaluation. Automation of Electric Power Systems, 2011; 35(02): 51-55.

[2] Lin CH, Chen CS, Chuang HJ, Huang MY, Huang CW. An expert system for three-phase balancing of distribution feeders. IEEE Transactions on Power Systems, 2008; 23(3): 1488-1496.

[3] Siti MW, Dan VN, Jimoh AA, et al. Reconfiguration and load balancing in the LV and MV distribution networks for optimal performance. IEEE Transactions on Power Delivery; 2007, 22(4):2534-2540.

[4] Hooshmand RA, Soltani S. Fuzzy optimal phase balancing of radial and meshed distribution networks using BF-PSO algorithm. IEEE Transactions on Power Systems; 2012, 27(1):47-57.

[5] Sun HJ, Peng CH, Yu TF. A three-phase equilibrium reconfiguration strategy for distribution network. Power System Technology; 2014, 38(03): 789-794.

[6] Xin YC, Li GQ, Wang CB. Compensation control of reactive power and three-phase unbalance load based on the method of sequence component. Power System Protection and Control; 2014, 42(14): 72-78.

[7] Peng H, Huang YN, Wang MH. Three-phase asymmetric load compensation in distribution system. Electric Power Automation Equipment; 2002(01): 32-34.

[8] Otto RA, Putman TH, Gyugyi L. Principles and applications of Static, thyristor-controlled shunt compensator. Power Apparatus \& Systems IEEE Transactions on; 1978, 97(5): 1935 -1945.

[9] Miller TJE. Reactive Power Control in Electric System. New York: John Willey\&Sons, 1982.

[10] Akagi H, Kanazawa Y, Naba A. Generalized theory of the instantaneous reactive power in three-phase circuits. Proc. JIEEIPEC. Tokyo, 1983: 1375-1386. 